\title{
Leiomiosarcoma escrotal con metástasis óseas
}

\author{
Diz Rodríguez MR*, Vírseda Chamorro M*, Ramírez García JR**, Merino Royo E**, \\ Moreno Reyes A***, Paños Lozano P*, ***. \\ *Servicio de Urología. Hospital Central de la Defensa. Madrid. **Servicio de Anatomía-patológica. \\ Hospital Central de la Defensa. Madrid. ***Servicio de Urología. Hospital Beata Maria de los Ángeles. \\ Madrid.
}

Actas Urol Esp. 2006; 30 (6): 638-640

\section{RESUMEN}

LEIOMIOSARCOMA ESCROTAL CON METÁSTASIS ÓSEAS

Se presenta un caso de leiomiosarcoma de escroto en un paciente de 87 años que debuta como una lesión en hemiescroto izquierdo indolora, y de un año de evolución. En el momento del diagnóstico se observa la presencia de metástasis óseas. Se realiza una revisión de la literatura comprobándose la rareza de este tipo de lesión (sólo se han descrito 27 casos), y que a diferencia de otro tipo de leiomiosarcomas, su evolución agresiva no es habitual en esta localización.

Palabras clave: Leiomiosarcoma. Escroto. Metástasis óseas.

\section{ABSTRACT \\ SCROTAL LEIOMYOSARCOMA WITH BONE METASTASIS}

A case of scrotum leiomyosarcoma is presented in a 87 year-old patient. It debuts as a painless lesion in left hemiescrotum, of one year of evolution. Bony metástasis were observed in the moment of the diagnosis. We carried out a literature revision proving the rarity of this lesion type (only 27 cases have been described), and that, contrary to another leiomiosarcomas type, their aggressive evolution is not habitual in this localization.

Keywords: Leiomyosarcoma. Scrotum. Bony Metastasis.

$\mathrm{E}$ 1 músculo liso sólo se encuentra en la piel de tres regiones: los folículos pilosos, la pared de los vasos y en los genitales externos masculinos (escroto y dartos peneano) y femeninos (vulva y tejido mamario) ${ }^{1}$.

Más del 95\% de los leiomiosarcomas paratesticulares se asientan en el cordón espermático o el epidídimo ${ }^{2}$, siendo su localización en la piel escrotal es excepcional.

Hasta la fecha se han descrito aproximadamente 27 leiomiosarcomas escrotales. La mayoría son de bajo grado, y la presencia de metástasis óseas en el momento del diagnóstico es excepcional $^{3}$.

Presentamos un caso de leiomiosarcoma testicular de alto grado en un varón de 84 años con metástasis óseas en el momento del diagnóstico.

\section{CASO CLÍNICO}

Paciente de 87 años de edad que acude a la consulta externa de urología por presentar desde hacía un año una tumoración en la cara externa del testículo izquierdo, sin síntomas urinarios acompañantes.

Entre sus antecedentes personales cabe reseñar: miocardiopatía dilatada de origen isquémico en tratamiento con antiagregantes plaquetarios, diabetes mellitus tipo II e HTA en tratamiento con un diurético de asa.

A la exploración, el paciente presenta en la zona externa de su hemiescroto izquierdo una tumoración de color blanquecino de aproximadamente dos centímetros, sólida, bien delimitada y no adherida a planos profundos. Bajo anestesia local se procedió a la extirpación de la tumoración 
testicular, estando ésta bien capsulada y fácilmente extirpable por su superficialidad y la ausencia de invasión a planos profundos. En el acto quirúrgico llamó la atención la gran vascularización de la tumoración. En el fondo del lecho quirúrgico el aspecto de la lesión era rigurosamente normal.

En el estudio anatomo-patológico se describe una tumoración de 2,6 × 2,0 x 1,5 cm de consistencia gomosa, color blanco grisáceo y aspecto nodular. Microscópicamente se apreciaba una neoformación maligna de estirpe mesenquimal con diferenciación muscular lisa constituida por fascículos entrelazados de células con citoplasma eosinófilo y núcleos fusiformes, con áreas de gran polimorfismo nuclear y abundantes mitosis (10 a 12 mitosis/10 campos de aumento). La tumoración presenta positividad para Vimentina, Desmina, Actina y Ki-67(positividad del $25 \%$ de los núcleos tumorales). La exéresis fue completa, aunque el margen libre de tumor resulto escaso en profundidad (Fig. 1).

Se realizó estudio de extensión mediante TAC abdomino-pélvica sin evidencia de extensión local, aunque se observó una imagen hipoecoica en polo superior de testículo izquierdo avascular compatible con proceso inflamatorio.

La gammagrafía ósea mostró una hipercaptación en hemipelvis derecha muy sugerente de infiltración ósea metastásica (Fig. 2).

\section{DISCUSIÓN}

El leiomiosarcoma escrotal es una neoplasia maligna infrecuente que se origina a partir de las fibras musculares del dartos.

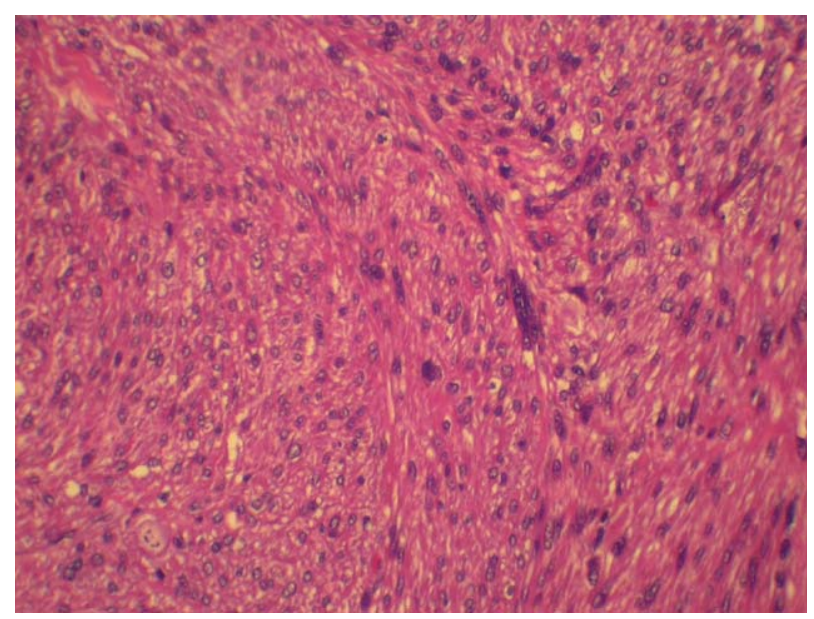

FIGURA 1. Proliferación de células fusiformes de núcleo pleomórfico, con gran cantidad de mitosis.

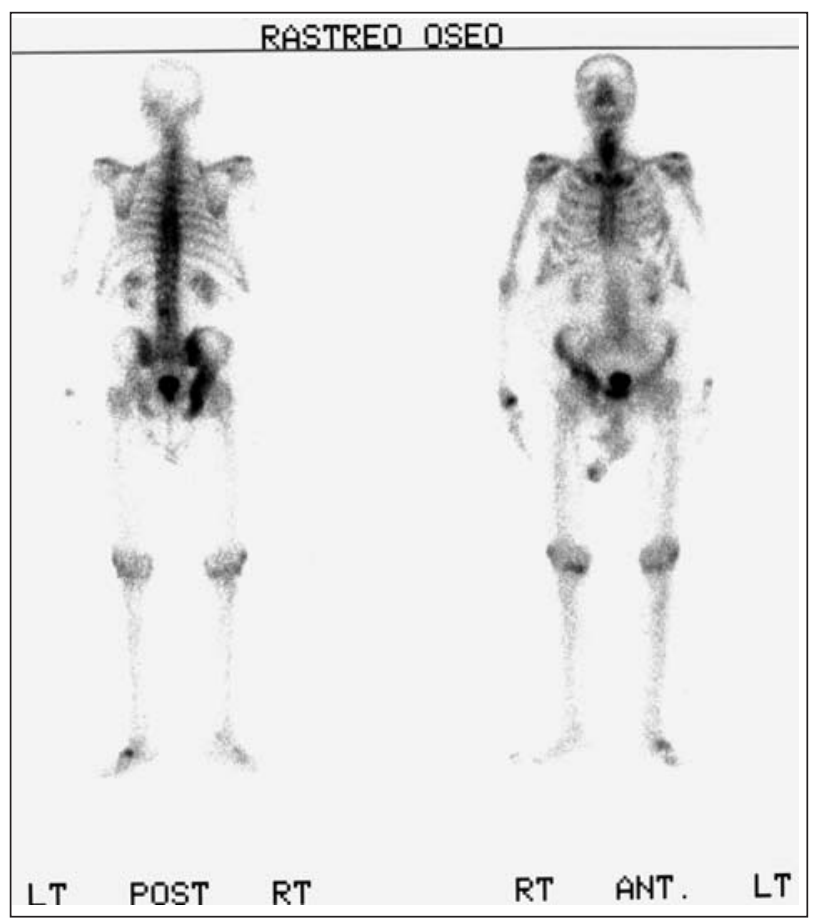

FIGURA 2. Gammagrafía ósea en la que se observa intensa hipercaptación del radiotrazador a nivel de rama isquiática derecha.

La mayor parte de las tumoraciones escrotales paratesticulares, a diferencia de las tumoraciones intratesticulres, son benignas. Sólo el 27\% de estas tumoraciones son malignas, siendo la mayoría de estirpe mesenquimatosa (rabdomiosarcomas, leiomiosarcomas o liposarcomas) ${ }^{4}$.

En la literatura en español sólo se han descrito tres casos de leiomiosarcoma escrotal ${ }^{5,7}$.

La edad media de aparición de los leiomiosarcomas escrotales es de 57 años con un rango entre los entre 34 y 86 años; nuestro paciente se encuentra incluido en el limite superior de dicho rango.

No se ha descrito una mayor incidencia en cuanto a la aparición de este tipo de tumores en uno u otro testículo siendo en nuestro caso el testículo izquierdo el afecto.

El leiomiosarcoma de escroto se presenta clínicamente como una tumoración sólida no adherida generalmente a planos profundos e indolora. Este aspecto de benigno es por lo que su diagnostico se puede demorar como en nuestro caso que el paciente acudió a consultas externas de nuestro centro un año después de la aparición de la tumoración. 
El tamaño medio de la tumoración en el momento del diagnóstico varía entre 2 y $9 \mathrm{~cm}$, con una media de $5 \mathrm{~cm}^{8}$. En nuestro caso las medidas de la tumoración eran de 26 × 20 × $15 \mathrm{~cm}$. Encontrándose bien capsulada ${ }^{9}$.

La presencia de fibras musculares lisas en su interior es la característica histológica fundamental de este tipo de tumores. El origen muscular del leiomiosarcoma lo confirma la inmunorreactividad a la vimentina, desmina y actina como ocurrió en nuestro caso ${ }^{8-10}$.

$\mathrm{Su}$ diagnóstico es exclusivamente anatomopatológico, no existiendo ninguna exploración complementaria que nos oriente en cuanto a su naturaleza. En el caso de los tumores de este tipo pero paratesticulares el diagnóstico ecográfico es difícil pero suelen corresponder a imágenes sólidas de gran volumen ${ }^{11}$

El pronostico del leiomiosarcoma depende fundamentalmente de tres aspectos; la profundidad de la lesión, el índice mitótico del tumor y de la aparición de metástasis a distancia. En nuestro caso, el paciente presentó dolores óseos a los seis meses del diagnóstico que fueron tratados sintomáticamente. Debido a la avanzada edad del paciente y a su morbilidad asociada, se decidió no realizar ningún tratamiento adyuvante.

La evolución de los leiomiosarcomas de las cubiertas genitales es diferente a la del resto de los leiomiosarcomas, siendo en general más benigna $^{12}$. Sin embargo, un $2 \%$ de los leiomiosarcomas paratesticulares recidivan localmente y se han descrito hasta un 29\% de metástasis linfáticas o viscerales (8\%). En nuestro caso presentó metástasis óseas en la hemipelvis derecha.

El tratamiento de este tipo de tumores se basa fundamentalmente en la extirpación local de la tumoración acompañado de un estrecho seguimiento. En el caso de los leiomiosarcomas de cordón espermático, algunos autores opinan que la orquiectomía local debe complementarse con radioterapia para evitar recidivas ${ }^{13}$.

\section{REFERENCIAS}

1. Flotte TJ, Bell DA, Sidhu GS, Plair CM. Leiomyosarcoma of the dartos muscle. J Cutan Pathol. 1981;8(1):69-74.

2. Echenique JE, Tully S, Tickman R, Walther MM, O'Brien DP, Judd RL. A 37-pound scrotal leiomyosarcoma: a case report and literature review. J Urol. 1987;138(5):12451246.

3. Ulbright T, Amin M, Young R. Atlas of Tumor Pathology. AFIP: Bethesda, 1997.

4. Jayaram N, Ramaprasad AV, Chethan M, Sujay RP. Tumours and tumour-like conditions of the para-testicular region-a study of morphological features. Indian $\mathrm{J}$ Pathol Microbiol. 1998;41(3):287-295.

5. Rodriguez-Parets JO, Silva Abuin J, Abad Hernandez M, Tinajas Saldana A, Martin Rodriguez A, Garcia Macias C, et al. Leiomioma atípico de escroto. A propósito de un caso. Actas Urol Esp. 1998;22(7):613-615.

6. Sanchez Bernal C, Navarro Martinez N, Vazquez Navarrete S. Leiomiosarcoma escrotal. A propósito de un caso. Actas Urol Esp. 1991;15(5):463-464.

7. Moreno A, Lamarca J, Garcia J. Leiomiosarcoma escrotal. Med Cutan Ibero Lat Am. 1984;12(5):387-389.

8. Fisher C, Goldblum JR, Epstein JI, Montgomery E. Leiomyosarcoma of the paratesticular region: a clinicopathologic study. Am J Surg Pathol. 2001;25(9):1143-1149.

9. Newman PL, Fletcher CD. Smooth muscle tumours of the external genitalia: clinicopathological analysis of a series. Histopathology 1991;18(6):523-529.

10. Desai SR, Angarkar NN. Leiomyosarcoma of the scrotum. Indian J Pathol Microbiol. 2003;46(2):212-213.

11. Woodward PJ, Schwab CM, Sesterhenn IA. From the archives of the AFIP: extratesticular scrotal masses: radiologicpathologic correlation. Radiographics. 2003;23(1): 215240.

12. Newman PL, Fletcher CD. Smooth muscle tumours of the external genitalia: clinicopathological analysis of a series. Histopathology. 1991;18(6):523-529.

13. Fagundes MA, Zietman AL, Althausen AF, Coen JJ, Shipley WU. The management of spermatic cord sarcoma. Cancer. 1996;77(9):1873-1876.

Dr. M. Vírseda Chamorro

E-mail bgmeli@terra.es

(Trabajo recibido 27 de mayo de 2005) 